\title{
Synthesis and Properties of gem-(Difluorocyclopropyl)amine
}

\section{Derivatives of Bicyclo[n.1.0]alkanes}

\author{
Ireneusz Nowak, John F. Cannon, and Morris J. Robins* \\ Department of Chemistry and Biochemistry, Brigham Young University, Provo, Utah \\ 84602-5700 \\ morris_robins@byu.edu
}

\section{Supporting Information}

Pages S2-S5: Experimental section, procedures, characterization data

Pages S6-S9: ${ }^{13} \mathrm{C}$ NMR spectra of $\mathbf{2 b}-\mathbf{2} \mathbf{i}$

Page S10: ${ }^{1} \mathrm{H}$ and ${ }^{13} \mathrm{C}$ NMR spectra of the 4c 2,4-dinitrophenylhydrazone

Page S11: ${ }^{1} \mathrm{H}$ and ${ }^{13} \mathrm{C}$ NMR spectra of the 4d 2,4-dinitrophenylhydrazone

Page S12: X-Ray crystal structure of $\mathbf{2} \mathbf{b} \bullet \mathrm{HCl}$

Page S13: X-Ray crystal structure of $\mathbf{2} \mathbf{c} \bullet \mathrm{HCl}$

Page S14: X-Ray crystal structure of $\mathbf{2 d} \cdot \mathbf{H B r}$

Page S15: X-Ray crystal structure of $\mathbf{2 e}$

Page S16: X-Ray crystal structure of $\mathbf{2} \cdot \bullet \cdot H C l$ 


\section{Experimental Section}

CAUTION! Organomercury compounds are extremely toxic. Avoid skin contact, use heavy-duty protective gloves, and use all other safety precautions for handling of highly toxic materials! General: Uncorrected melting points were determined with a Hoover capillary apparatus. ${ }^{1} \mathrm{H}$ (300 or $500 \mathrm{MHz}),{ }^{13} \mathrm{C}$ (75 or $125 \mathrm{MHz}$ ), and ${ }^{19} \mathrm{~F}$ (288 MHz) NMR spectra were recorded in $\mathrm{CDCl}_{3}$ unless noted. Electron impact mass spectra were obtained at $70 \mathrm{eV}$. High-resolution mass spectra (MS) were determined with EI or FAB [glycerol, thioglycerol, or p-nitrobenzyl alcohol (NBA)]. All chemicals and solvents were of reagent quality. All enamines, $\mathbf{1}$, were made by the procedure of Stork et al., ${ }^{1}$ and were used immediately after distillation.

General Procedure: Powdered NaI (5.6 g, $37.2 \mathrm{mmol})$ was stirred and heated at $170{ }^{\circ} \mathrm{C}$ for $1 \mathrm{~h}$ under vacuum in a flask $(250 \mathrm{~mL})$ equipped with a Teflon valve, and the flask was allowed to cool to ambient temperature. A solution of enamine $\mathbf{1 f}(2.0 \mathrm{~g}, 9.3 \mathrm{mmol})$ in dried THF (10 mL) and then a solution of $\mathrm{Hg}\left(\mathrm{CF}_{3}\right)_{2}(3.15 \mathrm{~g}, 9.3 \mathrm{mmol})$ in dried THF $(10 \mathrm{~mL})$ were injected through a septum under an atmosphere of $\mathrm{N}_{2}$. The reaction mixture was heated in an oil bath at $70{ }^{\circ} \mathrm{C}$ for $80 \mathrm{~min}$ and cooled to ambient temperature. Volatiles were concentrated, and the mixture was deposited on a column of silica gel. Chromatography (EtOAc/hexanes, 1:20) gave the difluorocyclopropylamine $\mathbf{2 f}(2.2 \mathrm{~g}, 89 \%)$ as a pale-yellow oil. This material was dissolved in $\mathrm{MeOH}(10 \mathrm{~mL})$, and conc. $\mathrm{HCl} / \mathrm{H}_{2} \mathrm{O}(3 \mathrm{~mL})$ was added with stirring. Volatiles were evaporated in vacuo. A purified sample of $\mathbf{2 f} \bullet \mathrm{HCl}$ was obtained by chromatography (EtOAc then $\mathrm{MeOH}$ ) followed by recrystallization $\left(\mathrm{EtOH} / \mathrm{Et}_{2} \mathrm{O}\right)$.

\section{Characterization Data:}

7,7-Difluoro-1-(morpholin-4-yl)bicyclo[4.1.0]heptane (2b). Colorless oil: ${ }^{1} \mathrm{H}$ NMR $\delta$ 1.15-1.82 (m, 8H), 1.84-2.02 (m, 1H), 2.57-2.62 (m, 4H), 3.61-3.70 (m, 4H); ${ }^{3} \mathrm{C}$ NMR $\delta 15.1$, 17.0, 20.4, 21.3, $25.0(\mathrm{t}, J=8.8 \mathrm{~Hz}), 46.2(\mathrm{t}, J=9.9 \mathrm{~Hz}), 48.0,66.8,116.5(\mathrm{dd}, J=285.3,293.7$ $\mathrm{Hz}) ;{ }^{19} \mathrm{~F}$ NMR $\delta 141.9$ (d, $\left.J=151.7 \mathrm{~Hz}, 1 \mathrm{~F}\right), 142.9$ (dd, $\left.J=15.0,151.7 \mathrm{~Hz}, 1 \mathrm{~F}\right) .2 \mathrm{~b} \bullet \mathrm{HCl} \cdot \mathrm{H}_{2} \mathrm{O}$ : mp 122-124 ${ }^{\circ} \mathrm{C}(\mathrm{THF})$; EI-MS m/z 217 (4\%, $\left.\mathrm{M}^{+}\right)$, 166, 91, 87; HRMS Calcd for $\mathrm{C}_{11} \mathrm{H}_{17} \mathrm{~F}_{2} \mathrm{NO}$ : 217.1278, Found: 217.1277.

8,8-Difluoro-1-(morpholin-4-yl)bicyclo[5.1.0]octane (2c). Colorless oil: ${ }^{1} \mathrm{H}$ NMR $\delta$ 1.06-2.10 (m, 10H), 2.23-2.30 (m, 1H), 2.64-2.82 (m, 4H), 3.54-3.69 (m, 4H); ${ }^{13} \mathrm{C}$ NMR $\delta 23.1$ $(\mathrm{d}, J=4.6 \mathrm{~Hz}), 23.4(\mathrm{~d}, J=4.6 \mathrm{~Hz}), 28.2,29.0,32.5,33.3(\mathrm{t}, J=8.0 \mathrm{~Hz}), 49.8,53.6(\mathrm{t}, J=9.2$ 
$\mathrm{Hz}), 67.3,116.5(\mathrm{dd}, J=287.6,296.8 \mathrm{~Hz}) ;{ }^{19} \mathrm{~F}$ NMR $\delta 141.9(\mathrm{dd}, J=15.0,145.2 \mathrm{~Hz}, 1 \mathrm{~F}), 142.9$ (d, $J=145.3 \mathrm{~Hz}, 1 \mathrm{~F}) .2 \mathrm{c} \cdot \mathrm{HCl}: \mathrm{mp} 163-165^{\circ} \mathrm{C}$ (dec.) $\left(\mathrm{EtOH}_{\mathrm{Et}} \mathrm{O}\right)$; EI-MS $\mathrm{m} / z 231\left(63 \%, \mathrm{M}^{+}\right)$, 216, 202, 188, 180 (100), 152; HRMS Calcd for $\mathrm{C}_{12} \mathrm{H}_{19} \mathrm{~F}_{2} \mathrm{NO}$ : 231.1435, Found: 231.1433.

9,9-Difluoro-1-(morpholin-4-yl)bicyclo[6.1.0]nonane (2d). Colorless oil: ${ }^{1} \mathrm{H}$ NMR $\delta$ 1.24-1.76 (m, 11H), 1.90-1.96 (m, 1H), 2.07-2.11 (m, 1H), 2.71-2.86 (m, 4H), 3.59-3.70 (m, $4 \mathrm{H}) ;(\mathrm{d}, J=3.1 \mathrm{~Hz}) ;{ }^{13} \mathrm{C}$ NMR $\delta 20.7,22.2,25.2,25.7,26.1,27.5,29.2(\mathrm{t}, J=7.5 \mathrm{~Hz}), 49.0,50.4$ $(\mathrm{t}, J=8.8 \mathrm{~Hz}), 67.8,115.9(\mathrm{t}, J=293.7 \mathrm{~Hz}) ;{ }^{19} \mathrm{~F}$ NMR $\delta 140.7$ (br d, $\left.J=151.7 \mathrm{~Hz}, 1 \mathrm{~F}\right), 145.4$ (d, $J=151.7 \mathrm{~Hz}, 1 \mathrm{~F}) . \mathbf{2 d} \cdot \mathrm{HBr}: \mathrm{mp} 150-155^{\circ} \mathrm{C}$ (dec.) (EtOH/THF); FAB-MS (glycerol) $m / z 246$ $\left(100 \%,[\mathrm{M}+\mathrm{H}]^{+}\right)$; HRMS Calcd for $\mathrm{C}_{13} \mathrm{H}_{22} \mathrm{~F}_{2} \mathrm{NO}: 246.1669$, Found: 246.1650 .

2,3-Benzo-7,7-difluoro-1-(morpholin-4-yl)bicyclo[4.1.0]heptane (2e). Crystals: mp 125-127 ${ }^{\circ} \mathrm{C}$ (toluene); ${ }^{1} \mathrm{H}$ NMR (CD $\left.\mathrm{OD}\right) \delta$ 1.51-1.59 (m, 2H), 2.16-1.26 (m, 1H), 2.18-1.36 (m, 1H), 2.46-2.54 (m, 1H), 2.64-2.80 (m, 3H), 2.97-3.06 (m, 2H), 3.30-3.32 (m, 1H), 3.63-3.78 (m, 4H), 7.17-7.21 (m, 1H), 7.24-7.31 (m, 2H), 7.40-7.44 (m, 1H); ${ }^{13} \mathrm{C}$ NMR $\delta 20.3$, 28.5, $30.1(\mathrm{t}, J=9.5 \mathrm{~Hz}), 49.5(\mathrm{t}, J=11.4 \mathrm{~Hz}), 49.6,67.2,116.4(\mathrm{dd}, J=288.4,296.8 \mathrm{~Hz})$, $126.2,127.3,127.6,128.5,129.9,140.6 ;{ }^{19} \mathrm{~F} \mathrm{NMR}\left(\mathrm{DMSO}-d_{6}\right) \delta 135.8(\mathrm{~d}, J=143.1 \mathrm{~Hz}, 1 \mathrm{~F})$, $136.6(\mathrm{dd}, J=15.0,143.1 \mathrm{~Hz}, 1 \mathrm{~F})$; FAB-MS (NBA) $m / z, 266\left(75 \%,[\mathrm{M}+\mathrm{H}]^{+}\right), 264(100 \%)$; HRMS Calcd for $\mathrm{C}_{15} \mathrm{H}_{18} \mathrm{~F}_{2} \mathrm{NO}$ : 266.1356, Found: 266.1371. 2e• $\mathrm{HCl}$ (prepared by the general procedure) lost $\mathrm{HCl}$ upon heating to give mp and MS data identical to that of $\mathbf{2 e}$.

8,8-Difluoro-1-[( $N$-benzyl)( $N$-methyl)amino]bicyclo[5.1.0]octane (2f). Colorless oil: ${ }^{1} \mathrm{H}$ NMR $\delta 1.10-2.10(\mathrm{~m}, 10 \mathrm{H}), 2.28(\mathrm{~s}, 3 \mathrm{H}), 2.34-2.38(\mathrm{~m}, 1 \mathrm{H}), 3.73(\mathrm{~d}, J=13.2 \mathrm{~Hz}, 1 \mathrm{H}), 3.87$ $(\mathrm{d}, J=13.2 \mathrm{~Hz}, 1 \mathrm{H}), 7.19-7.31(\mathrm{~m}, 5 \mathrm{H}) ;{ }^{13} \mathrm{C} \mathrm{NMR} \delta 23.7(\mathrm{~d}, J=6.1 \mathrm{~Hz}), 23.7(\mathrm{~d}, J=4.5 \mathrm{~Hz})$, 28.1, 29.2, 32.8, $34.3(\mathrm{t}, J=8.4 \mathrm{~Hz}), 37.7,54.8(\mathrm{t}, J=9.2 \mathrm{~Hz}), 59.2,117.9(\mathrm{dd}, J=288.4,296.0$ $\mathrm{Hz}), 126.8,128.06,128.12,139.7 ;{ }^{19} \mathrm{~F} \mathrm{NMR}\left(\mathrm{Me}_{2} \mathrm{CO}-d_{6}\right) \delta 140.4(\mathrm{dd}, J=17.1,145.2 \mathrm{~Hz}, 1 \mathrm{~F})$, $142.2(\mathrm{~d}, J=145.2 \mathrm{~Hz}, 1 \mathrm{~F}) . \mathbf{2 f} \bullet \mathrm{HCl}: \mathrm{mp} 152-154{ }^{\circ} \mathrm{C}(\mathrm{dec}).(\mathrm{EtOH} / \mathrm{THF}) ; \mathrm{FAB}-\mathrm{MS}$ (glycerol) $m / z 266\left(100 \%,[\mathrm{M}+\mathrm{H}]^{+}\right), 185,174$; HRMS Calcd for $\mathrm{C}_{16} \mathrm{H}_{22} \mathrm{~F}_{2} \mathrm{~N}: 266.1720$, Found: 266.1719.

8,8-Difluoro-1-( $N, N$-dibenzylamino)bicyclo[5.1.0]octane (2g). Colorless oil: ${ }^{1} \mathrm{H}$ NMR

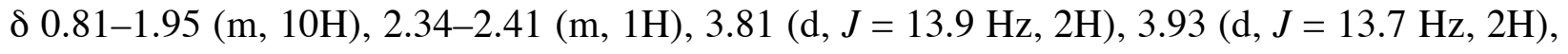
7.14-7.32 (m, 10H); ${ }^{13} \mathrm{C}$ NMR $\delta 23.7(\mathrm{~d}, J=3.8 \mathrm{~Hz}), 26.1,27.1,28.0,32.5,33.4(\mathrm{t}, J=8.4 \mathrm{~Hz})$, $53.8(\mathrm{t}, J=9.5 \mathrm{~Hz}), 56.5,117.4(\mathrm{t}, J=294.5 \mathrm{~Hz}), 126.8,127.9,128.8,139.8 ;{ }^{19} \mathrm{~F}$ NMR $\delta 136.1$ $(\mathrm{dd}, J=17.1,147.4 \mathrm{~Hz}, 1 \mathrm{~F}), 143.6$ (d, $J=147.4 \mathrm{~Hz}, 1 \mathrm{~F}) .2 \mathrm{~g} \cdot \mathrm{HCl}$ : Orange oil: FAB-MS 
(glycerol) $m / z 342\left(80 \%,[\mathrm{M}+\mathrm{H}]^{+}\right), 340,288(100 \%)$; HRMS Calcd for $\mathrm{C}_{22} \mathrm{H}_{26} \mathrm{~F}_{2} \mathrm{~N}: 342.2033$, Found: 342.2032.

8,8-Difluoro-1-[(N-methyl)(N-phenyl)amino]bicyclo[5.1.0]octane (2h). Pale-yellow oil: ${ }^{1} \mathrm{H}$ NMR $\delta 1.18-1.29$ (m, 1H), 1.36-1.45 (m, 1H), 1.50-1.61 (m, 3H), 1.65-1.73 (ddd, $J=$ 7.3, 10.7, 17.6 Hz, 1H), 1.91-2.01 (m, 2H), 2.15-2.23 (m, 1H), 2.28-2.37 (m, 1H), 3.08 (s, 3H), 6.68-6.78 (m, 3H), 7.23-7.28 (m, 2H); ${ }^{13} \mathrm{C}$ NMR $\delta 23.5,26.7,26.8,28.5,32.1(\mathrm{t}, J=9.1 \mathrm{~Hz})$, 32.2, 36.8, $51.5(\mathrm{t}, J=10.3 \mathrm{~Hz}), 112.6,116.81$ (dd, $J=293.0,294.5 \mathrm{~Hz}), 116.85,128.8,147.8$; ${ }^{19}$ F NMR $\delta 135.9(\mathrm{dd}, J=17.1,147.4 \mathrm{~Hz}, 1 \mathrm{~F}), 143.4(\mathrm{~d}, J=147.4 \mathrm{~Hz}, 1 \mathrm{~F}) ; \mathrm{FAB}-\mathrm{MS}$ (thioglycerol) $m / z 252\left(100 \%,[\mathrm{M}+\mathrm{H}]^{+}\right)$; HRMS Calcd for $\mathrm{C}_{15} \mathrm{H}_{20} \mathrm{~F}_{2} \mathrm{~N}$ : 252.1564, Found: 252.1564 .

8,8-Difluoro-1-( $N, N$-dipentylamino)bicyclo[5.1.0]octane (2i). Colorless oil: ${ }^{1} \mathrm{H}$ NMR $\delta$ $0.89(\mathrm{t}, J=7.1 \mathrm{~Hz}, 6 \mathrm{H}), 1.10-1.92(\mathrm{~m}, 21 \mathrm{H}), 2.02-2.09(\mathrm{~m}, 1 \mathrm{H}), 2.13-2.19$ (m, 1H), 2.54-2.72 $(\mathrm{m}, 4 \mathrm{H}) ;{ }^{13} \mathrm{C}$ NMR $\delta 14.1,22.7,23.8(\mathrm{~d}, J=3.8 \mathrm{~Hz}), 24.6,27.2,28.9,29.6,32.7,33.3(\mathrm{t}, J=8.4$ $\mathrm{Hz}), 51.0,52.9,53.8(\mathrm{t}, J=8.7 \mathrm{~Hz}), 117.8(\mathrm{dd}, J=291.4,294.5 \mathrm{~Hz}) ;{ }^{19} \mathrm{~F}$ NMR $\left(\mathrm{DMSO}-\mathrm{d}_{6}\right) \delta$ 133.5 (dd, $J=18.2,141.0 \mathrm{~Hz}, 1 \mathrm{~F}), 141.4$ (d, $J=141.0 \mathrm{~Hz}, 1 \mathrm{~F}) .2 \mathrm{i} \cdot \mathrm{HBr}$ : Orange oil: FAB-MS (thioglycerol) $m / z 300\left(100 \%,[\mathrm{M}-\mathrm{H}]^{+}\right)$; HRMS Calcd for $\mathrm{C}_{18} \mathrm{H}_{32} \mathrm{~F}_{2} \mathrm{~N}$ : 300.2503, Found: 300.2517 .

Thermal Decomposition of 8,8-Difluoro-1-(morpholin-4-yl)bicyclo[5.1.0]octane (2c). A sample of $2 \mathrm{c}(2.10 \mathrm{~g}, 9.09 \mathrm{mmol})$ was heated at $170{ }^{\circ} \mathrm{C}$ for $24 \mathrm{~h}$ and treated as described for the conversion of $\mathbf{2 d} \rightarrow \mathbf{4 d}$ to give $\mathbf{4 c}$. Careful concentration of the solution of the somewhat volatile 2-fluorocyclooct-2-enone (4c), and its treatment with 2,4-dinitrophenylhydrazine (300 $\mathrm{mg}, 1.52 \mathrm{mmol})$ gave the purified derivative $(130 \mathrm{mg}$, $4 \%$ overall from $\mathbf{2 c}$ after recrystallisation from $\mathrm{CH}_{2} \mathrm{Cl}_{2} / \mathrm{EtOH}$ ) as lustrous, bright-orange plates: mp $186-188{ }^{\circ} \mathrm{C} ;{ }^{1} \mathrm{H} \mathrm{NMR} \delta 1.58-1.65(\mathrm{~m}$, 2H), 1.69-1.75 (m, 2H), 1.90-1.96 (m, 2H), 2.46-2.52 (m, 2H), 2.84-2.89 (m, 2H), $5.89(\mathrm{dt}, J=$ 8.5, 24.4 Hz, 1H), 8.08 (d, $J=9.8 \mathrm{~Hz}, 1 \mathrm{H}), 8.35$ (dd, $J=2.4,9.8 \mathrm{~Hz}, 1 \mathrm{H}), 9.14$ (d, $J=2.4 \mathrm{~Hz}$, 1H), $11.41(\mathrm{~s}, 1 \mathrm{H}) ;{ }^{13} \mathrm{C}$ NMR $\delta 22.5,23.6(\mathrm{~d}, J=8.4 \mathrm{~Hz}), 23.7,25.4,26.3,113.2(\mathrm{~d}, J=23.7$ $\mathrm{Hz}), 117.1,123.2,130.1,138.7,144.8,149.3(\mathrm{~d}, J=18.3 \mathrm{~Hz}), 155.5(\mathrm{~d}, J=244.9 \mathrm{~Hz}) ;{ }^{19} \mathrm{~F} \mathrm{NMR}$

$\delta 111.3(\mathrm{~d}, J=23.5 \mathrm{~Hz}, 1 \mathrm{~F})$; FAB-MS (thioglycerol) $\mathrm{m} / \mathrm{z} 323\left(100 \%,[\mathrm{M}+\mathrm{H}]^{+}\right), 345(55 \%$, [M $+\mathrm{Na}]^{+}$); HRMS Calcd for $\mathrm{C}_{14} \mathrm{H}_{15} \mathrm{FN}_{4} \mathrm{O}_{4} \mathrm{Na}$ : 345.0975, Found: 345.0983.

\section{Thermal Decomposition of 9,9-Difluoro-1-(morpholin-4-yl)bicyclo[6.1.0]nonane}

(2d). Compound $2 \mathbf{d}(700 \mathrm{mg}, 2.86 \mathrm{mmol})$ was heated at $100{ }^{\circ} \mathrm{C}$ for $1 \mathrm{~h}$ in a glass tube equipped 
with a Teflon valve. The purple residue contained 2-fluoro-3-(4-morpholin-1-yl)-1,3cyclononadiene (3d) and 2-fluorocyclonon-2-enone (4d) in a 3:1 ratio (NMR). The residue was dissolved in $\mathrm{MeOH}(5 \mathrm{~mL})$ and stirred with $5 \% \mathrm{HCl} / \mathrm{H}_{2} \mathrm{O}(5 \mathrm{~mL})$ for $15 \mathrm{~min}$. Volatiles were removed in vacuo, and the residue was partitioned $\left(\mathrm{EtOAc} / \mathrm{H}_{2} \mathrm{O}\right)$. Volatiles were evaporated from the organic phase, and the residue was chromatographed (EtOAc/hexanes, 1:3) to give 4d (320 $\mathrm{mg}, 72 \%$ ) as a pale-yellow oil whose spectral data were in harmony with literature values, and a sample was converted into its 2,4-dinitrophenylhydrazone. Thus, 2,4-dinitrophenylhydrazine (430 mg, $2.18 \mathrm{mmol}$ ) was suspended in $\mathrm{MeOH}(8 \mathrm{~mL})$, and conc. $\mathrm{H}_{2} \mathrm{SO}_{4} / \mathrm{H}_{2} \mathrm{O}(0.9 \mathrm{~mL})$ was added. The resulting mixture was filtered with a cotton plug, and the filtrate was collected in a flask containing 4d (200 mg, $1.28 \mathrm{mmol})$ in $\mathrm{MeOH}(3 \mathrm{~mL})$. After $1 \mathrm{~h}$, filtration gave crystals that were recrystallized several times $\left(\mathrm{CH}_{2} \mathrm{Cl}_{2} / \mathrm{EtOH}\right)$ to give the purified derivative (200 $\mathrm{mg}, 48 \%$ ) as lustrous, bright-orange plates: $\mathrm{mp} 165-167{ }^{\circ} \mathrm{C} ;{ }^{1} \mathrm{H}$ NMR $\delta 1.50-1.64(\mathrm{~m}, 6 \mathrm{H}), 1.92-1.98$ (m, 2H), 2.38-2.46 (m, 2H), 2.78-2.86 (m, 2H), 5.72 (dt, $J=9.8,24.9 \mathrm{~Hz}, 1 \mathrm{H}), 8.07$ (d, $J=9.8 \mathrm{~Hz}$, $1 \mathrm{H}), 8.35(\mathrm{dd}, J=2.4,9.8 \mathrm{~Hz}, 1 \mathrm{H}), 9.14(\mathrm{~d}, J=2.4 \mathrm{~Hz}, 1 \mathrm{H}), 11.41(\mathrm{~s}, 1 \mathrm{H}) ;{ }^{13} \mathrm{C}$ NMR $\delta 22.3(\mathrm{~d}, J$ $=8.4 \mathrm{~Hz}), 23.4,25.10,25.13,26.2,27.9,112.8(\mathrm{~d}, J=23.7 \mathrm{~Hz}), 117.0,123.1,130.0,138.7$, 144.6, $149.8(\mathrm{~d}, J=22.1 \mathrm{~Hz}), 155.0(\mathrm{~d}, J=242.6 \mathrm{~Hz}) ;{ }^{19} \mathrm{~F}$ NMR $\delta 109.3$ (d, $\left.J=23.5 \mathrm{~Hz}, 1 \mathrm{~F}\right)$; EI-MS m/z $336\left(100 \%, \mathrm{M}^{+}\right.$], 319, 299; HRMS Calcd for $\mathrm{C}_{15} \mathrm{H}_{17} \mathrm{FN}_{4} \mathrm{O}_{4}$ : 336.1233, Found: 336.1241 .

(1) Stork, G.; Brizollara, A.; Landesman, H.; Szmuszkovich, J.; Terrell, R. J. Org. Chem. 1963, 85, 207-221.

(2) Blanco, L.; Rousseau, G. Bull. Soc. Chim. Fr. 1985, 3, 455-462. 

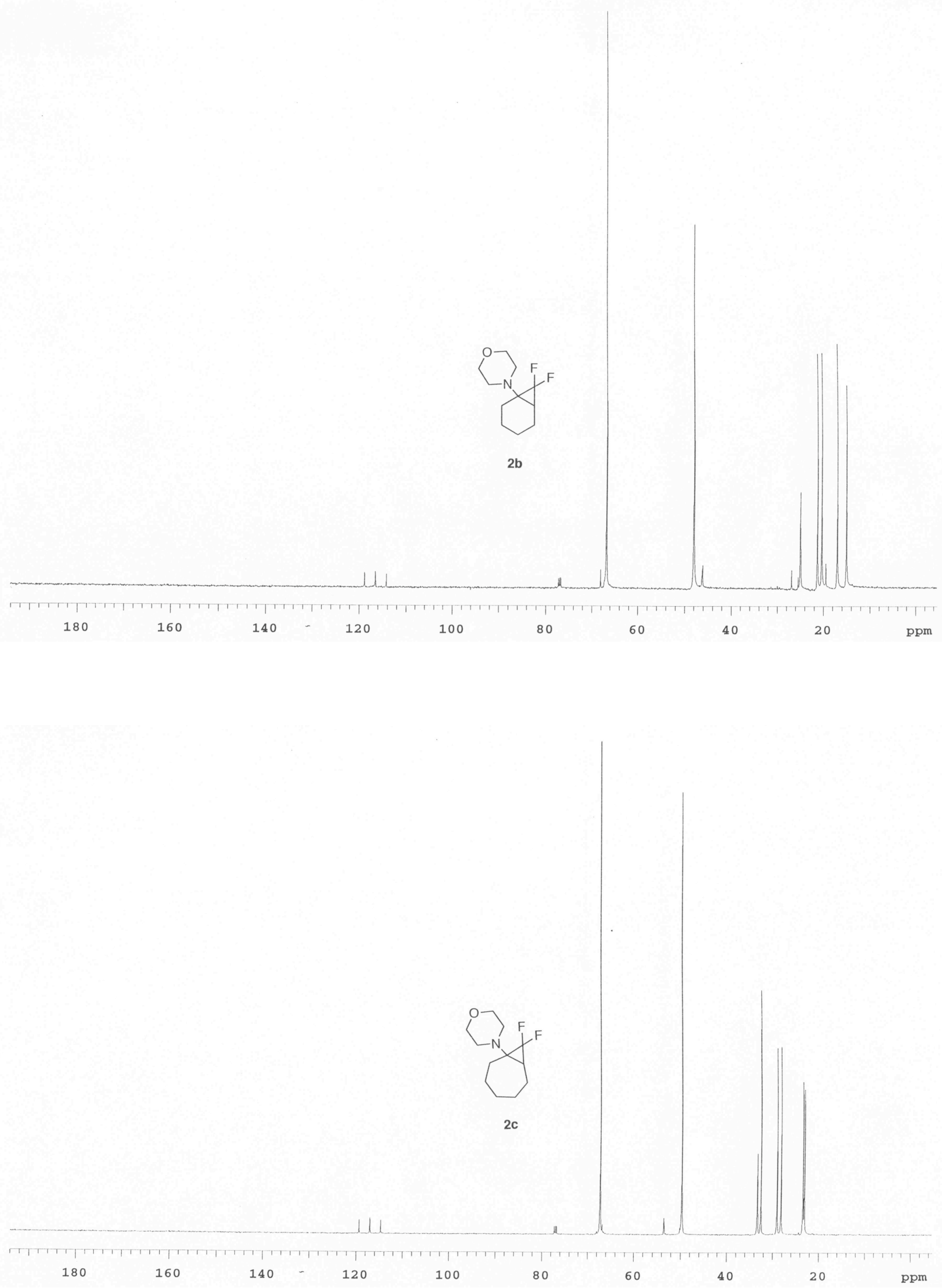

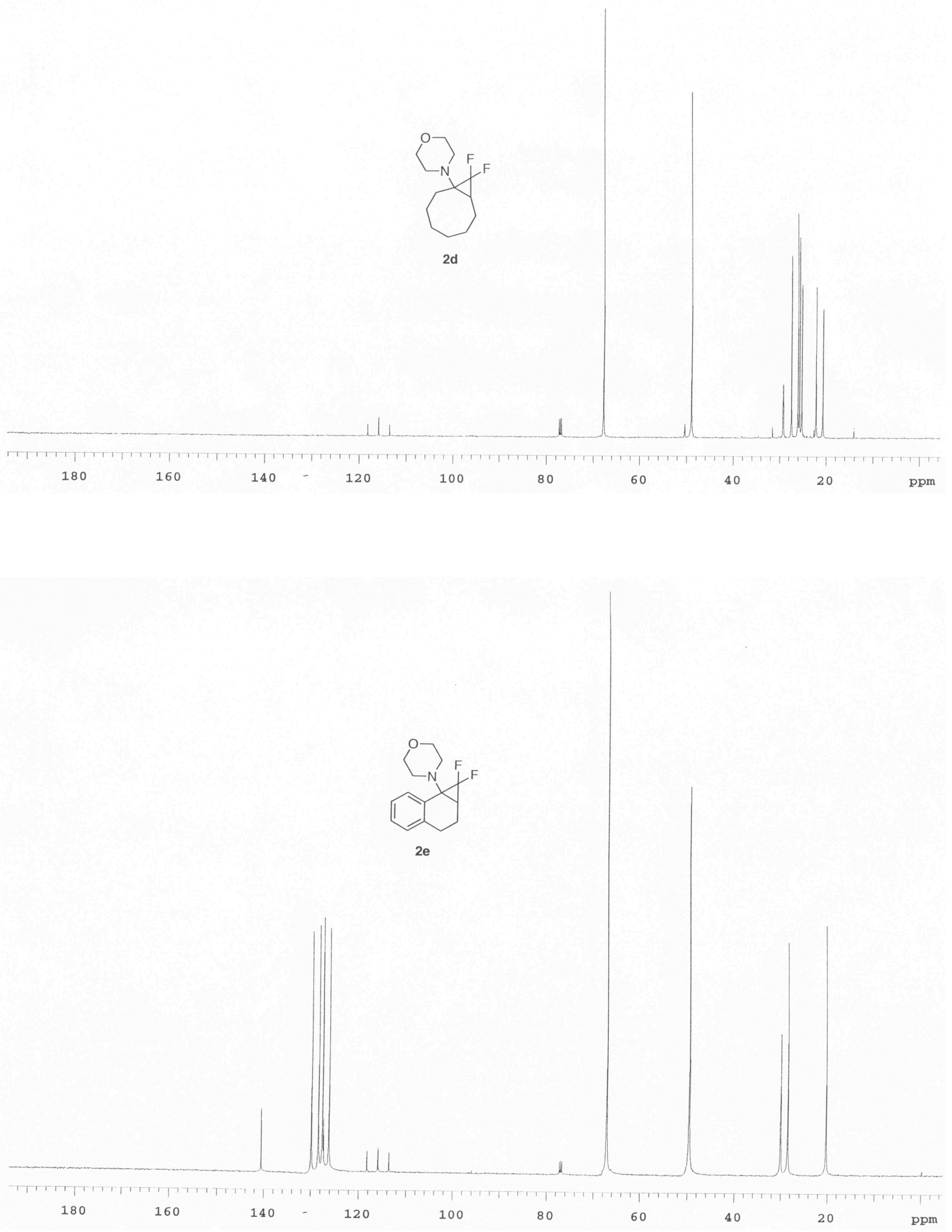

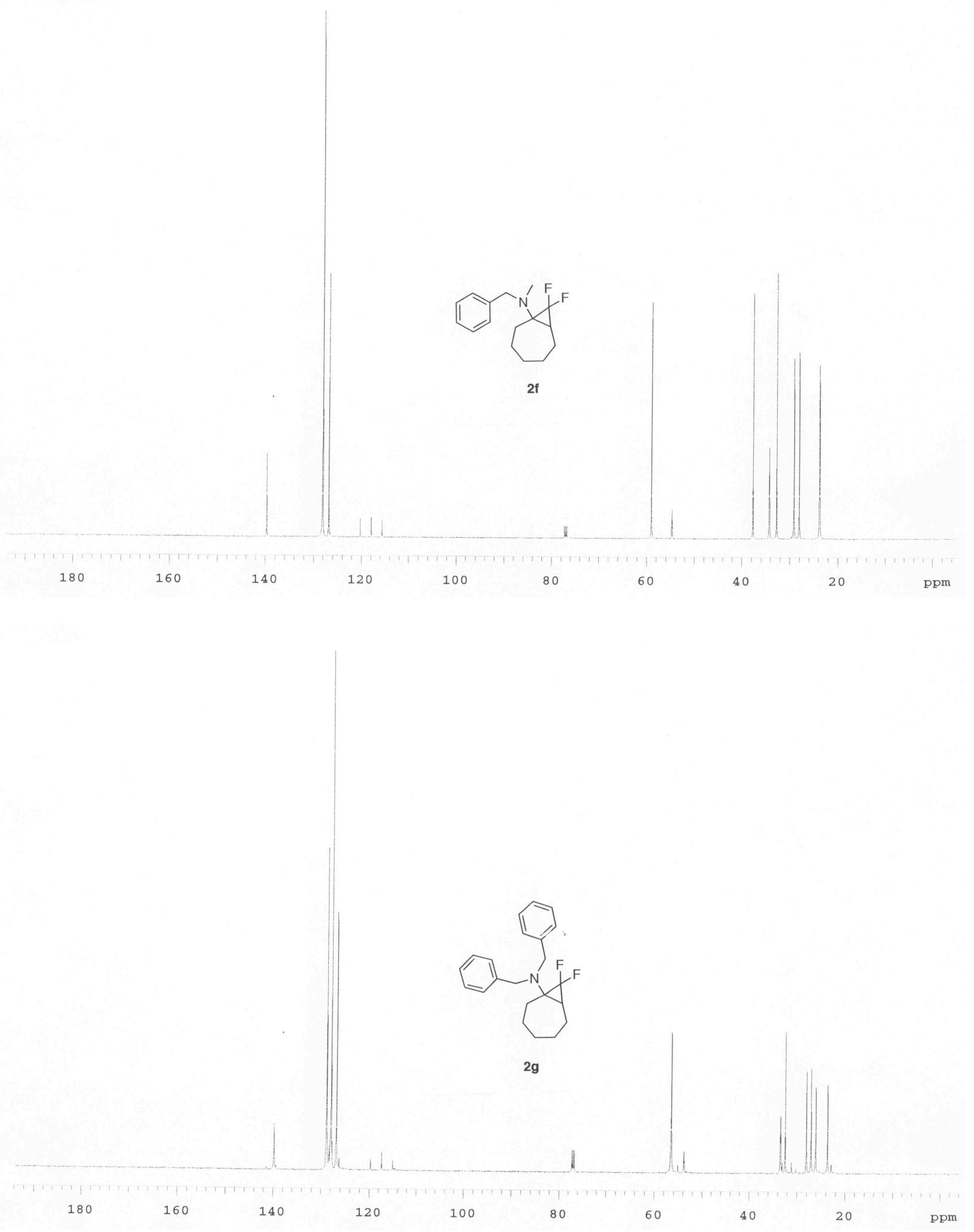

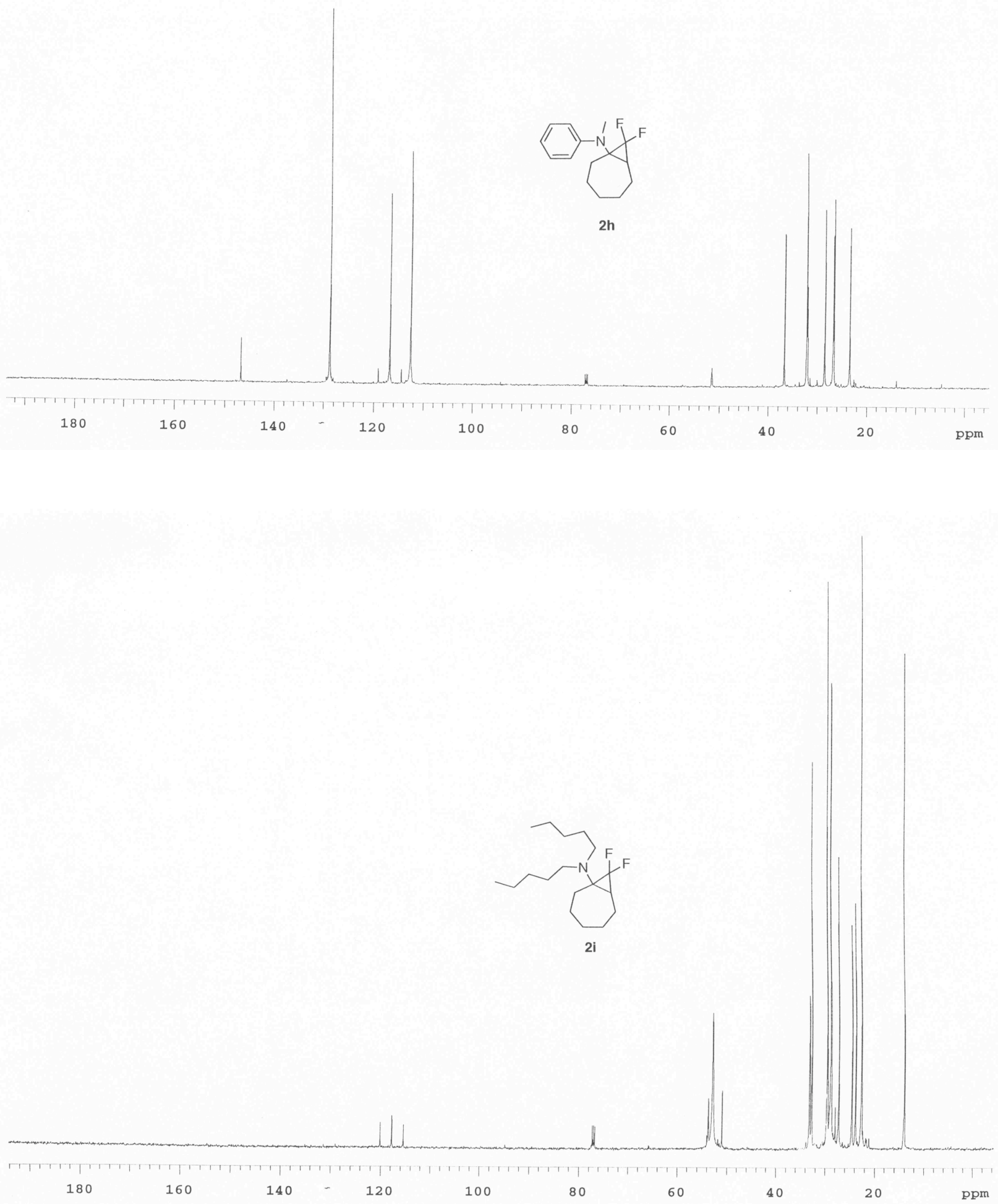
S10

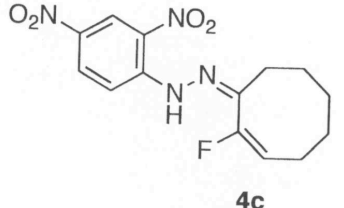

4c
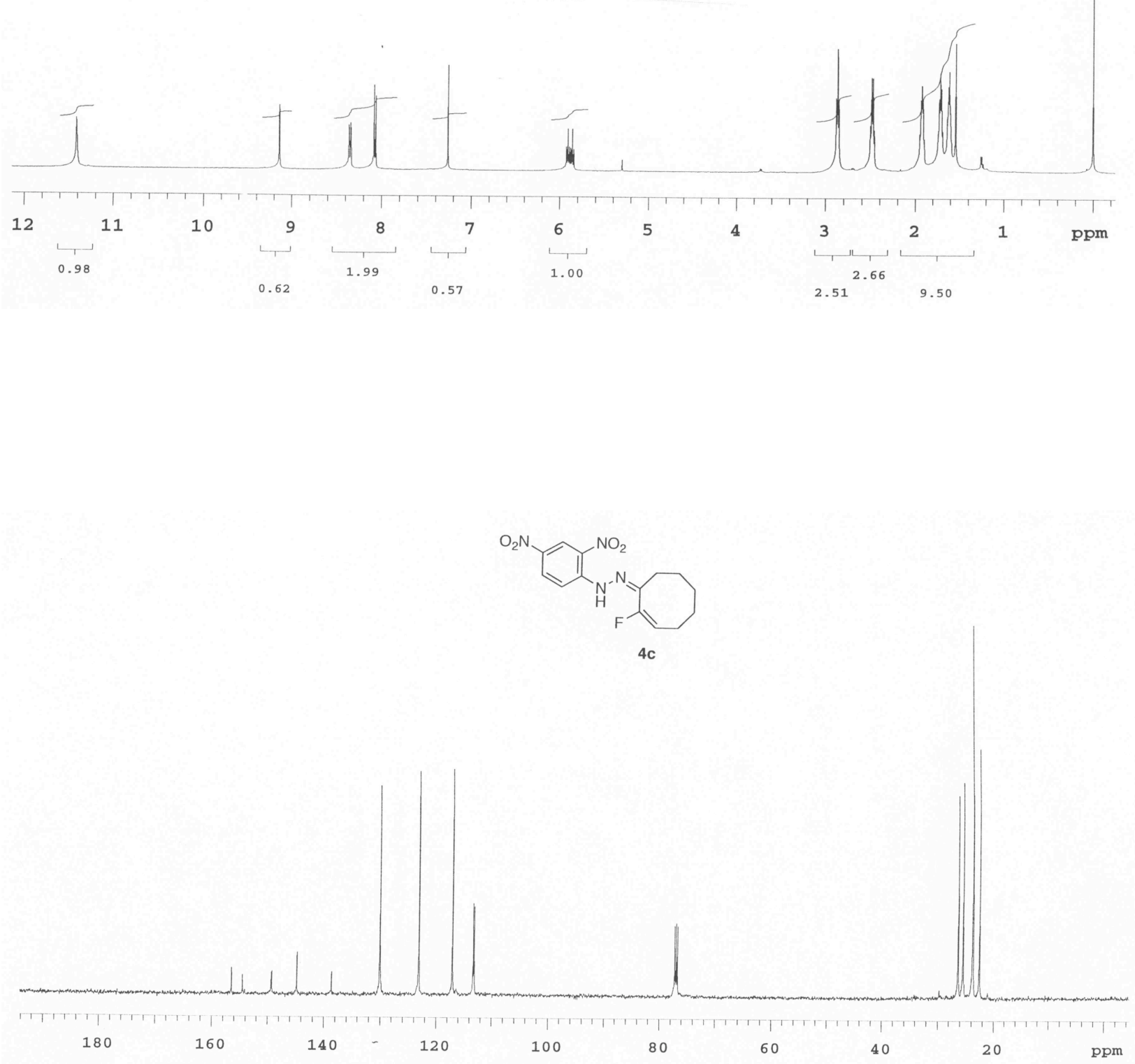

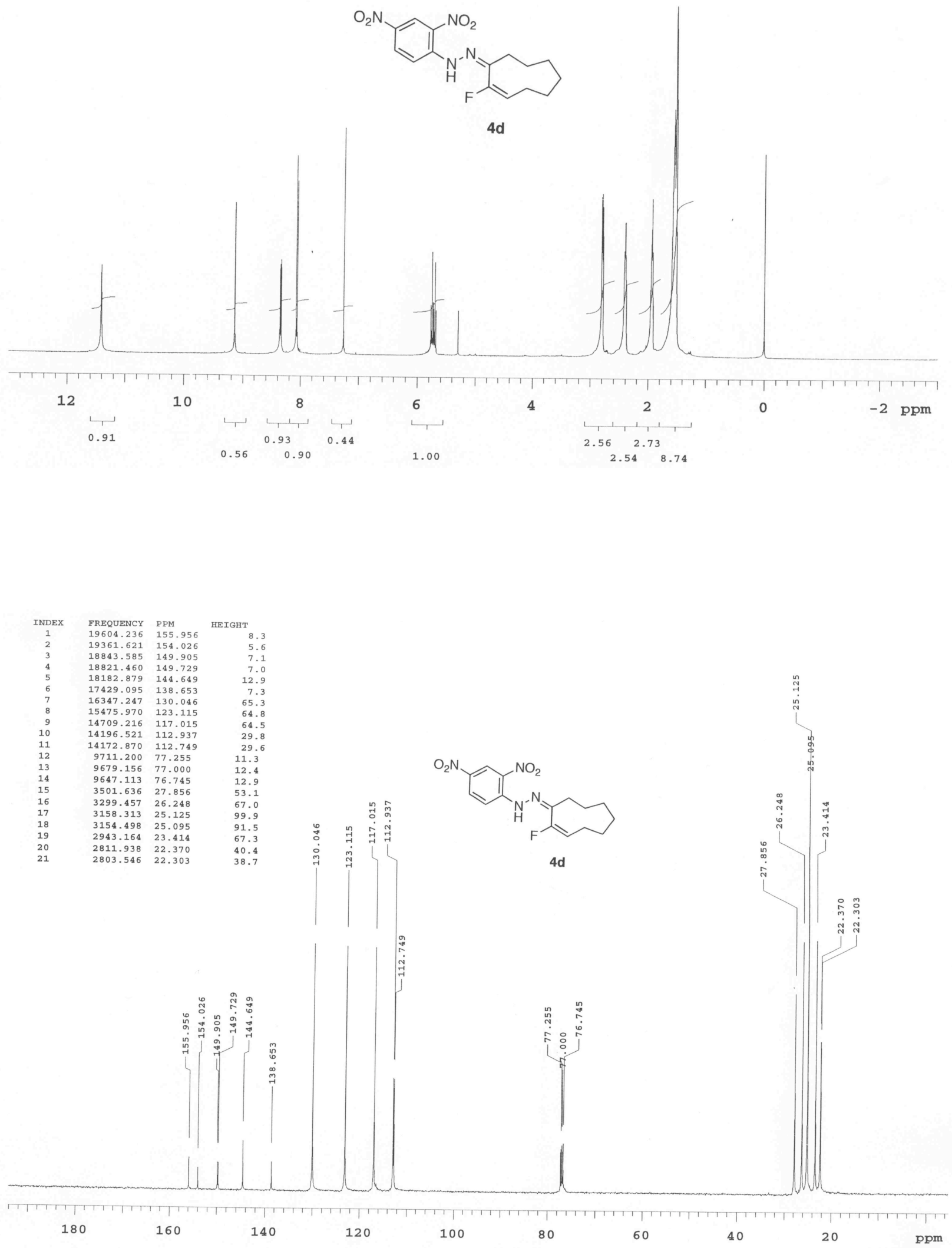


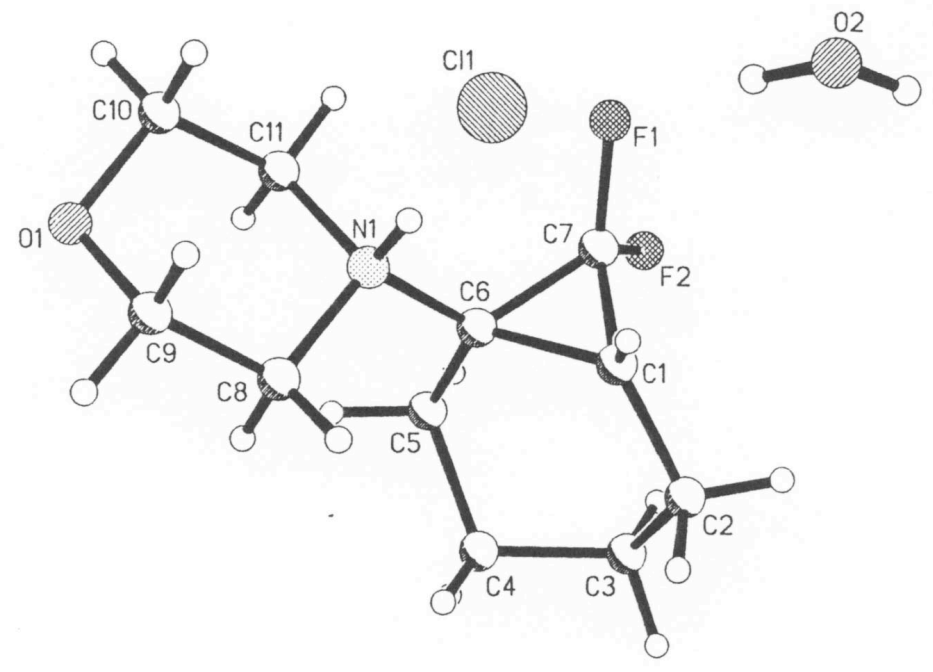

Figure S1. X-ray structure of $\mathbf{2} \mathbf{b} \mathrm{HCl}$ salt. 


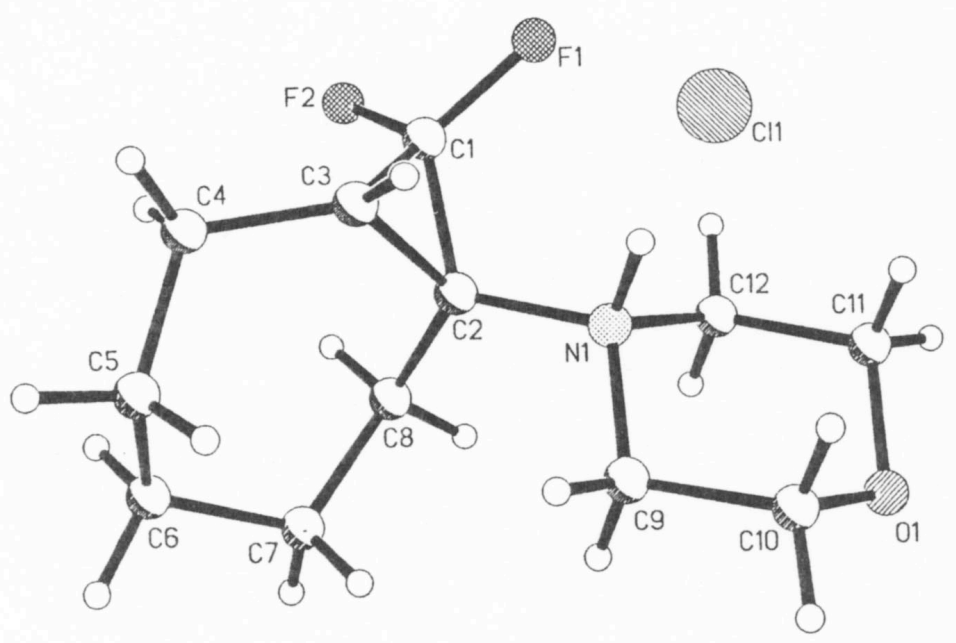

Figure S2. X-ray structure of $\mathbf{2} \mathbf{c ~} \mathrm{HCl}$ salt. 


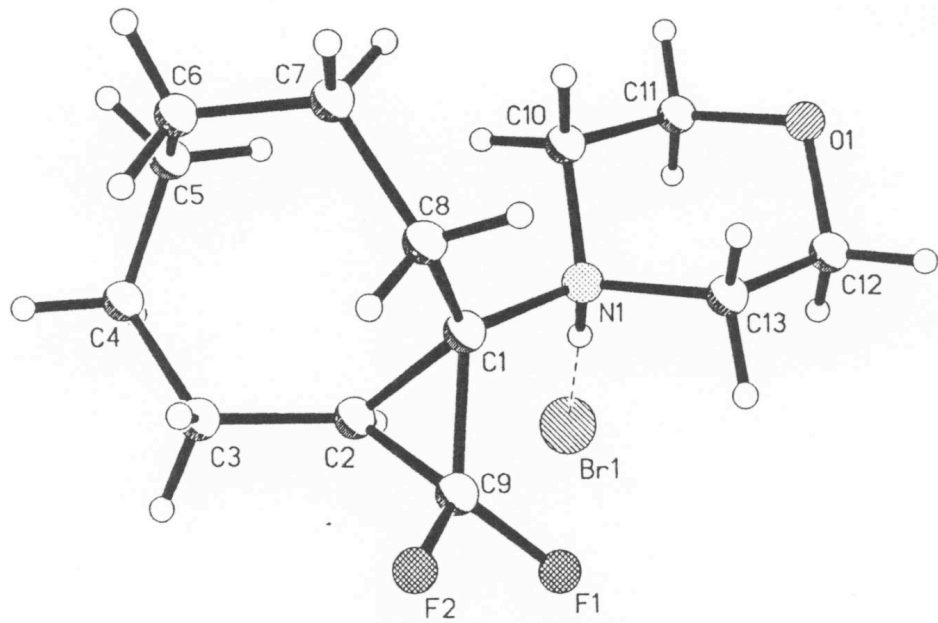

Figure S3. X-ray structure of $\mathbf{2 d} \mathrm{HBr}$ salt. 


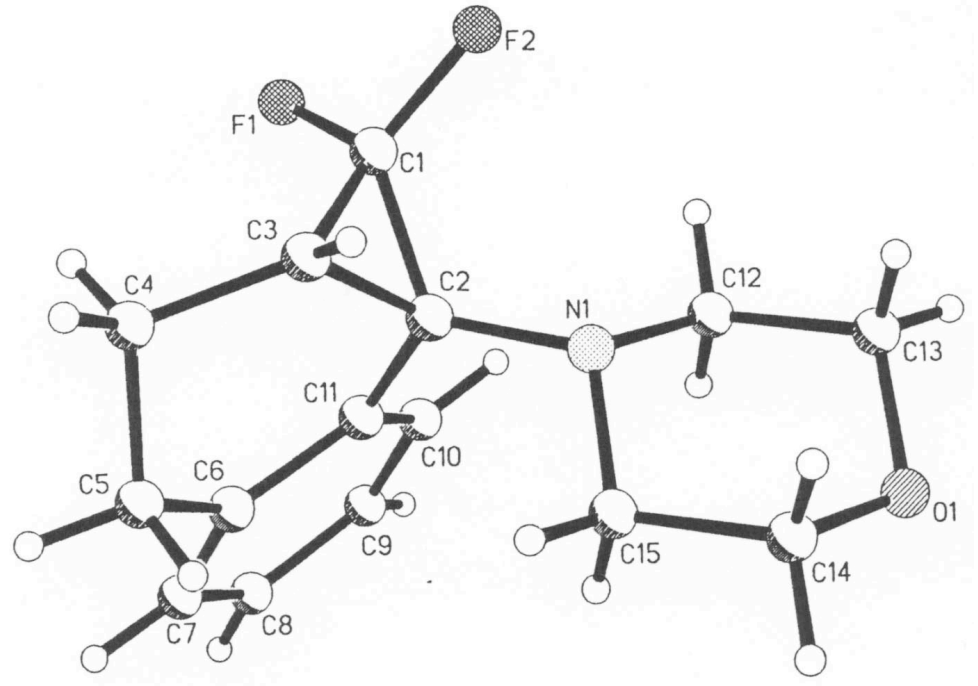

Figure S4. X-ray structure of $2 e$. 


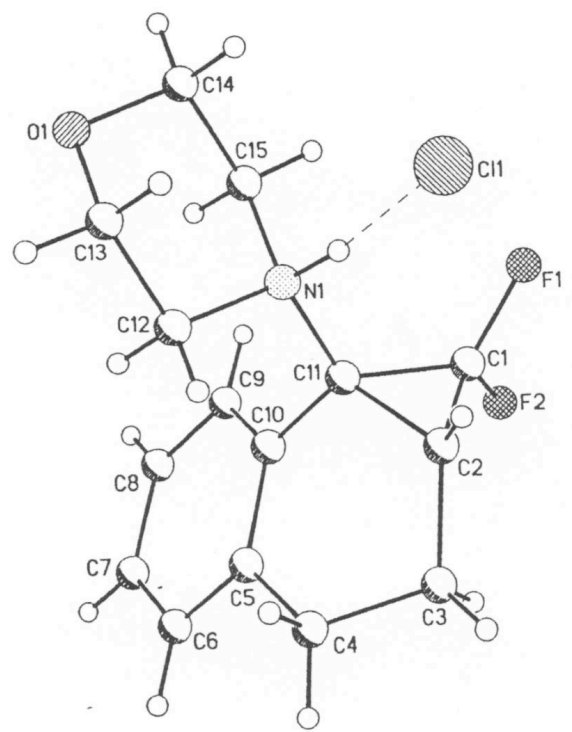

Figure S5. X-ray structure of $2 \mathrm{e} \mathrm{HCl}$ salt. 